\title{
Ac Conductivity and Dielectric Studies of Polypyrrole Copper Zinc Iron Oxide Nanocomposites
}

\author{
V.S. Shanthala ${ }^{1}$, S.N. Shobha Devi ${ }^{2}$, M. V. Murugendrappa ${ }^{3}$ \\ ${ }^{I}$ The Oxford College of Science,Department of Physics,Bangalore, Karnataka,India \\ ${ }^{2}$ Department of Physics, BMS College for Women, Bangalore, Karnataka, India \\ ${ }^{3}$ Department of Physics, BMS College of Engineering, Bangalore, Karnataka, India
}

\begin{abstract}
Polypyrrole and polypyrrole/copper zinc iron oxide $\left(\mathrm{CuZnFe}_{2} \mathrm{O}_{4}\right)$ nanocomposites are synthesized by in-situ polymerisation in different weight percentages using oxidation method. Dielectric and conductivity behaviours of Polymer nanocomposites of various composition were investigated using impedance spectroscopy at different temperatures and frequencies $(100 \mathrm{~Hz}-5 \mathrm{MHz})$. The dielectric properties of host polymer matrix have been improved by the addition of nanoparticles and are found to be highly temperature dependent. Both Dielectric constant and Dielectric loss decreased with increase in frequency for all composites, indicating a normal behaviour of dielectrics. Increase in dielectric constant and dielectric loss was observed with respect to temperature, which is attributed to increase in number of dipoles due to thermal energy. Dielectric relaxation is observed at low frequency range, relaxation time is calculated from the dielectric loss peaks. The highest conductivity was observed at $373 \mathrm{~K}$ for all nanocomposites. The significant increase in dielectric constant makes them a potential candidate for dielectrics in capacitors used for decoupling, timing, filtering, and many other functions.
\end{abstract}

Keywords: Polypyrrole, copper zinc iron oxide, nanocomposite, dielectric constant, conductivity, frequency

\section{Introduction}

Nanotechnology has high impact in creating new classes of materials with enhanced functionality and a wide range of applications. Polymer Nanocomposites belong to this class and form an exciting field of research. As they exhibit amazing combination of properties they find applications in EMI shielding, electronic devices, Microwave absorbers, sensors, rechargeable batteries etc;[1]. Conducting polymers are class of polymer that possess good tunable electrical conductivity. But they are chemically sensitive and have poor mechanical properti; on the other hand, nanomaterials exhibit good mechanical properties. Thus nanocomposites formed by blending conducting polymers with inorganic nanoparticles like oxides, possess all the good properties of both the constituents and hence enhanced utility thereof. The properties of nanocomposites of such kind are strongly dependent on concentration of polymer. The state of dispersion of nanoparticles in the polymeric matrix has often a large impact on the properties of polymers[2]. In polymer nanocomposites conductivity depends on various factors such as filler morphology, size, loading concentration, compactness and interfacial interaction. The dramatically larger chain-particle interface area in the case of nanocomposites makes effects appearing negligible in microcomposites very prominent in nanocomposites [24] Polypyrrole (Ppy) is one of the most attractive polymers due to its special transport properties, facile synthesis, higher conductivity and good environmental stability. Polypyrrole has the advantages real applications in batteries, electronic devices, optical switching devices, functional electrodes, sensors and so on.[5]. ]. Nano crystalline ferrites are materials of considerable interest due to their unique dielectric, magnetic and optical properties. Copper zinc iron oxide nano particles (copper zinc nano ferrites) are used in sensorsLPG sensing.[6] Copper zinc iron oxide nano particles of size $<100 \mathrm{~nm}$ are selected for our investigation.

In this paper we report $\mathrm{AC}$ conductivity and Dielectric properties of $\mathrm{PPy} / \mathrm{CuZnFe}{ }_{2} \mathrm{O}_{4}$ nanocomposites of different compositions.

\section{Experimental Details}

Chemicals - The monomer pyrrole, oxidising agent Ammonium persulphate, nanopowder Copper zinc iron oxide, acetone were purchased from Sigma Aldrich. All chemicals were of analytical grade and used as received without any further treatment.

\subsection{Synthesis of Polypyrrole}

PPy was synthesized by in-situ polymerization of monomer pyrrole in the presence of oxidising agent ammonium persulphate. $0.3 \mathrm{M}$ pyrrole taken in a round bottomed glass flask was placed in an ice tray mounted on a magnetic stirrer. $0.6 \mathrm{M}$ Ammonium persulphate was added drop wise using a burette to the above $0.3 \mathrm{M}$ pyrrole. The reaction was carried out for 5 hours under continuous stirring maintaining temperature of 0 to 5 
degree Celsius. The resulting precipitate was removed by filtration by suction. The polypyrrole powder thus obtained was then dried in a hot air oven and subsequently in a muffle furnace at a temperature of 100 degree Celsius. The yield was $2.15 \mathrm{gm}$, taken as $100 \mathrm{Wt} \%$ [7].

\subsection{Synthesis of Ppy/ Cuznfe ${ }_{2} \mathrm{o}_{4}$ nanocomposites.}

PPy $/ \mathrm{CuZnFe}{ }_{2} \mathrm{O}_{4}$ nanocomposites were synthesized by the same method. For $0.3 \mathrm{M}$ pyrrole solution, 0.215 gms (10 wt.\%) of $\mathrm{CuZnFe}_{2} \mathrm{O}_{4}$ was added and mixed thoroughly in a round bottomed flask. O.6M ammonium persulphate was added drop-wise with the help of burette to the above solution and the entire procedure is repeated to get $10 \mathrm{wt} \%$ composite. Similarly $0.430 \mathrm{~g}, 0.6 \mathrm{~g}, 0.860 \mathrm{~g}, 1.075 \mathrm{~g}$ of nanopowder is taken and the entire procedure is repeated to get 20,30,40 and $50 \mathrm{wt} \% \mathrm{PPy} / \mathrm{CuZnFe}_{2} \mathrm{O}_{4}$ nanocomposites.. The immiscibility of heterophases of the polymer and $\mathrm{CuZnFe}_{2} \mathrm{O}_{4}$ constituents has been resolved by ultrasonicating the mixture, which has ensured the homogeneous dispersion of nanoparticles in the polymer matrix.

\subsection{Preparation of Pellets:}

The pure polypyrrole and $\mathrm{PPy} / \mathrm{CuZnFe}_{2} \mathrm{O}_{4}$ nanocomposites are pressed in the form of pellets of diameter $10 \mathrm{~mm}$ and thickness 1 to $3 \mathrm{~mm}$, using hydraulic press by applying 10-12 tons of pressure. The pellets of the synthesized composites are coated with silver paste on either side of the surfaces to obtain better contacts for conductivity measurements[8].

\subsection{AC Conductivity Measurements}

An analysis of ac conductivity and dielectric properties of PPy nanocomposites has been carried out using impedance spectroscopy on application of a small a.c. signal across the sample (pellet) which is sandwiched between two stainless steel electrodes under spring pressure. Complex impedance parameters (i.e., capacitance, dissipation factor, impedance, Resistance and phase angles parameters) were measured with a computer-controlled impedance analyzer ((Wayn kerr 6500B) in the frequency range from $100 \mathrm{~Hz}-5 \mathrm{MHz}$ at different temperatures [9]. Using these parameters conductivity, dielectric constant and dielectric loss of the nanocomposites have been calculated. The Conductivity $(\sigma)$, dielectric constant $\left(\varepsilon^{\prime}\right)$ and dielectric loss $\left(\epsilon^{\prime \prime}\right)$ are calculated by using the relations,

$\sigma=\frac{t}{R a}$ where ' $\mathrm{R}$ ' is the resistance measured from LCR meter and ' $\mathrm{t}$ ' is the thickness

$\varepsilon^{\prime}=\frac{c_{p} t}{\epsilon_{0} a} \quad$ where ' $c_{p}$, is the capacitance measured from LCR meter and ' $\mathrm{t}$ ' is the thickness of pellet. And $\in_{0}$ is the absolute permittivity $=8.85 \times 10^{-12} \mathrm{~F} / \mathrm{m}$

' $a$ ' is the area of the sample $=\pi r^{2}$.

$\epsilon^{\prime \prime}=\varepsilon^{\prime} \tan \delta$

\section{Dielectric Properties:}

\section{Results and Discussion}

The conductivity behaviour of conducting polymer can be understood from dielectric analysis. The dielectric properties have been analyzed in terms of temperature, frequency and compositions, This analysis measures two fundamental electrical characteristic of materials: (1) the capacitive (insulating) nature, which represents its ability to store the electric charges and (2) The conductive nature, which represents its ability to transfer electronic charge. Through this analysis, the dielectric constant $\varepsilon^{\prime}$ and dielectric loss $\varepsilon^{\prime \prime}$ of a material can be determined.

\subsection{Dielectric Constant}

Fig.1. shows the variation of dielectric constant of nanocomposites with frequency at room temperature. It is observed from the figure that Nanocomposites with composition $40 \%$ show highest dielectric constant compare to the remaining composites, and $\varepsilon^{\prime}$ decreases with increase in frequency for all the nanocomposites which is the typical behaviour of dielectrics, At low frequency dipoles follow the field and we have $\varepsilon^{\prime}=\varepsilon_{\mathrm{s}}$, This results in a high value of dielectric constant. On the other hand, at high frequencies, dipoles begin to lag behind the field, periodic reversal of the electric field occurs so fast that there is no excess ion diffusion in the direction of the field and hence leading to the observed decrease in dielectric constant[10-11].

Fig.2a, 2b, 2c, 2d, 2e and 2f represents Frequency dependence of dielectric constant at different temperatures for nanocomposites with 10, 20,30, 40,50 Wt.\% and Pure PPy respectively.

The dielectric constant has a maxima at $300 \mathrm{~Hz}$ for nanocomposites of $10 \mathrm{wt} . \%$ at $303 \mathrm{~K}$. For $20 \mathrm{wt} . \%$ composites at higher temperature the peaks are observed at $700 \mathrm{~Hz}$ and $200 \mathrm{~Hz}$. The dielectric constant is constants at the other temperatures, for nanocomposites of $30 \%$ the peak is observed at $200 \mathrm{~Hz}$, a peak is observed at $1000 \mathrm{~Hz}$ for $40 \mathrm{wt} . \%$ at $100 \mathrm{k}$, a small peak is also seen at $200 \mathrm{~Hz}$ at $303 \mathrm{~K}$, for $50 \%$ no peaks are seen, 
but the dielectric constant is maximum at $100 \mathrm{~K}$, The variation is almost same as PPy, except that dielectric constant is large at $423 \mathrm{~K}$ for PPy.
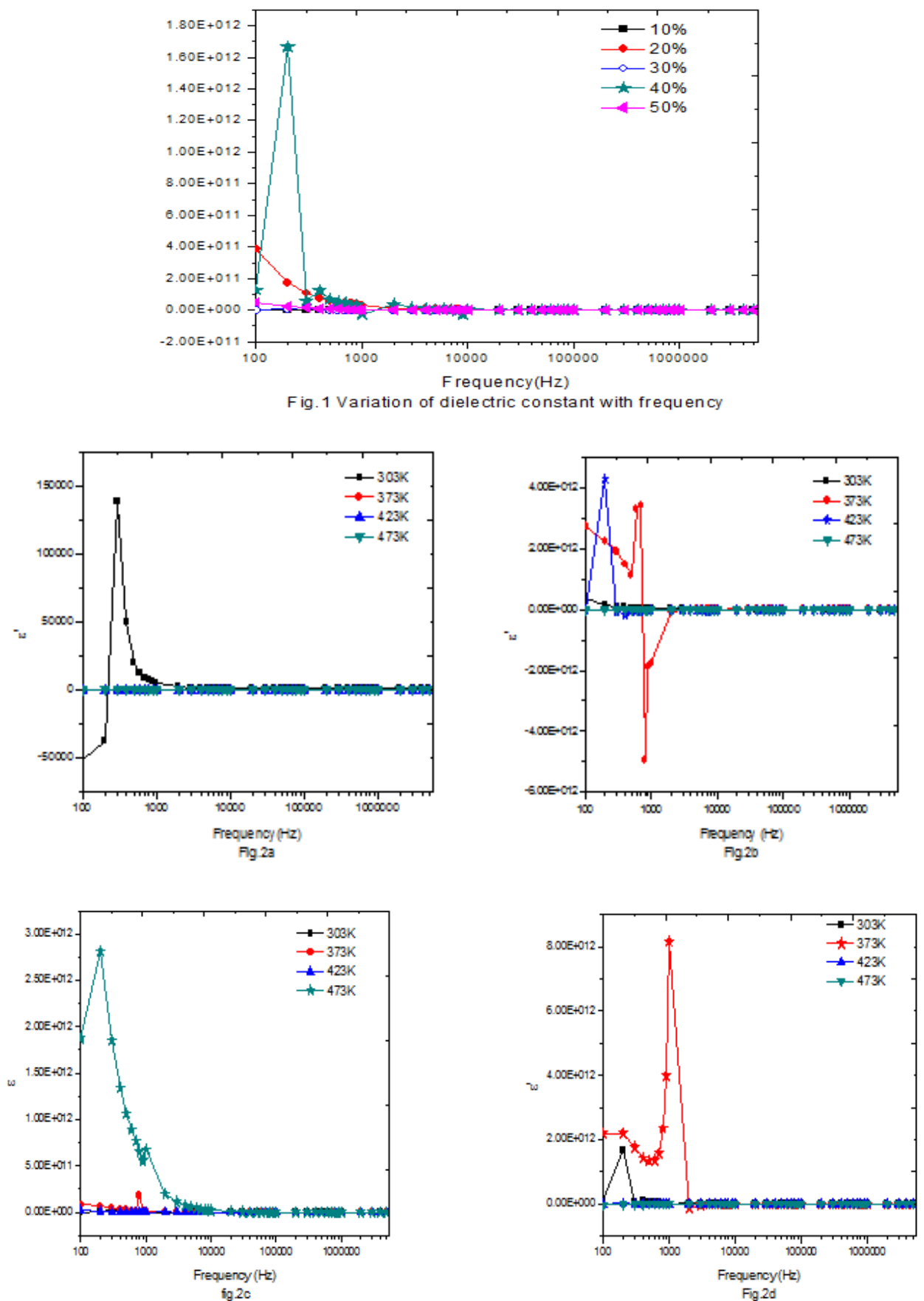

The dielectric constant increases with temperature for all nanocomposites except for nanocomposites of $10 \mathrm{wt} \%$. The increase in dielectric constant may be attributed to electric field which is accompanied by the frequencies. Such field will cause some ordering or orientation in samples, the molecular dipoles cannot orient themselves at low temperature, when the temperature is increased the dipole orientation is facilitated and dielectric constant increases. The strong frequency dispersion of permittivity is observed in the low frequency region followed by a nearly frequency independent behaviour above $10 \mathrm{KHz}$.The peak in the graph can be attributed to strong relaxation contribution leading to high dielectric constants and therefore electronic and ionic polarizablity[12].The negative dielectric constant observed at some frequencies for some wt.percentage is due 

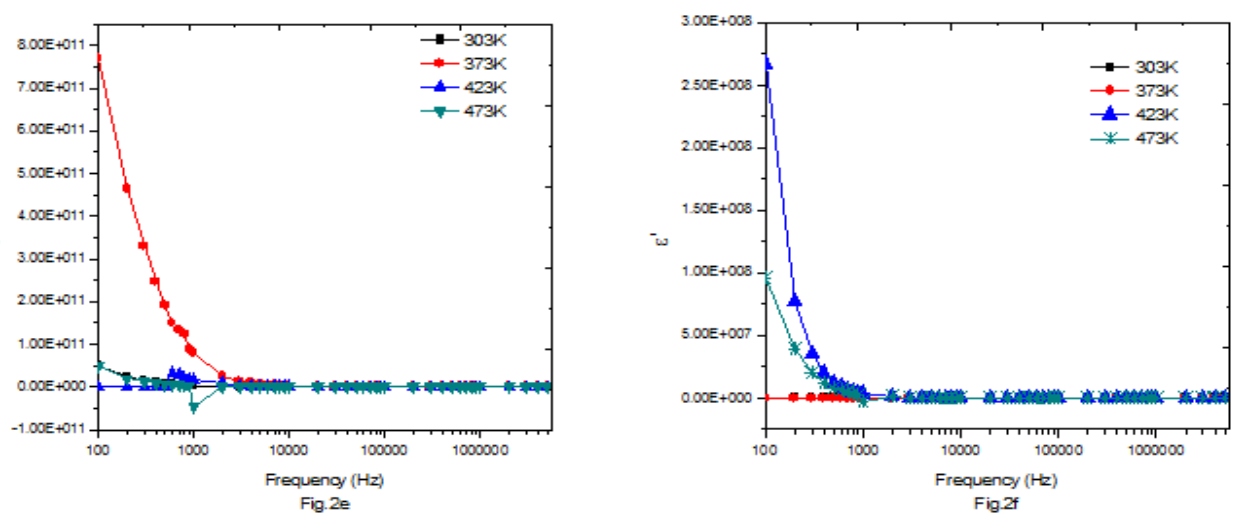

to its reduced plasma frequency and an induction effect. The magnitude of the negative dielectric constant and the resonance frequency are tunable by dopant type and doping composition.

\subsection{Dielectric Loss:}
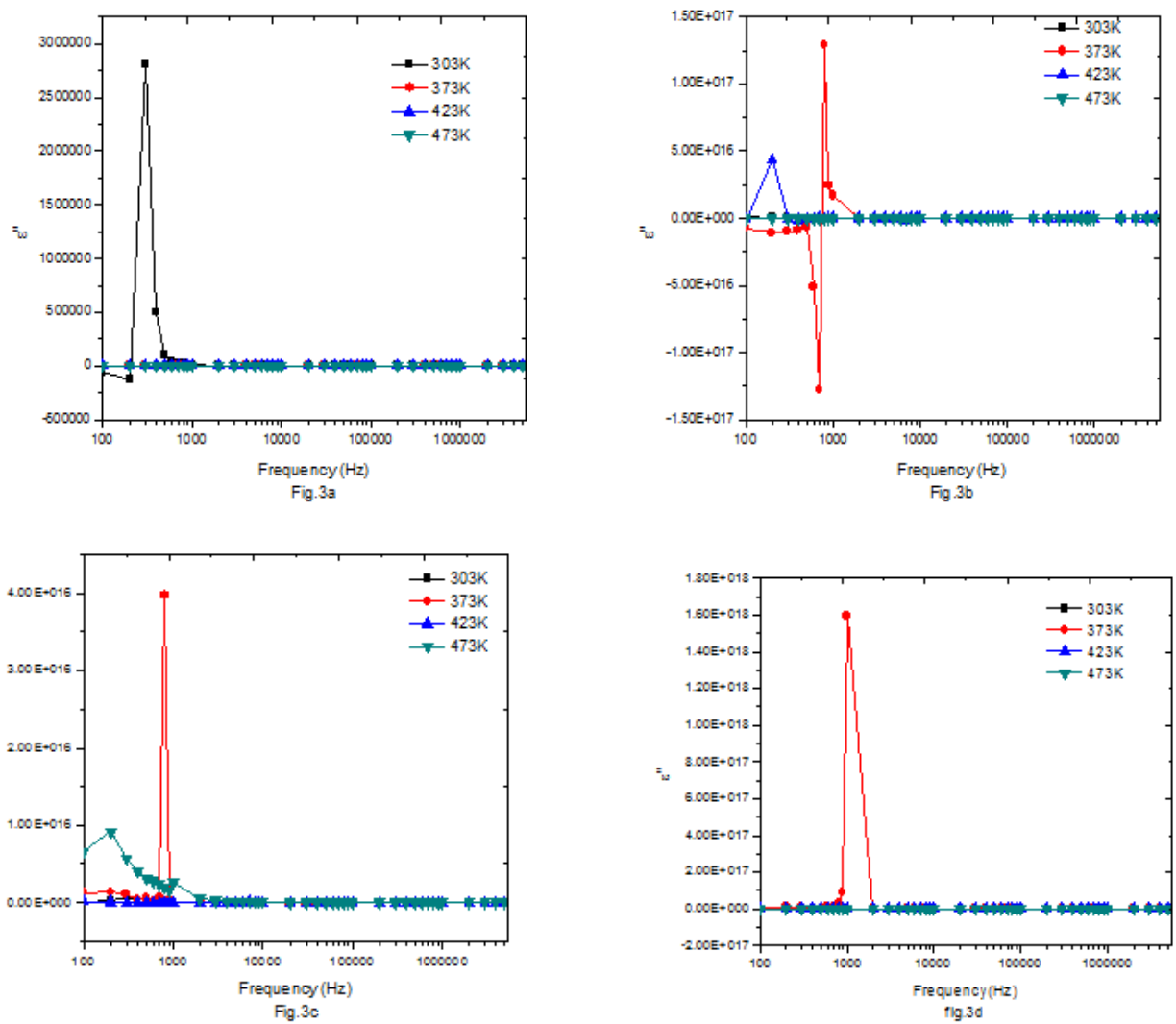

Fig.3. shows variation of dielectric loss with frequency which exhibits a dielectric loss peaks in the frequency range $300-100 \mathrm{~Hz}$. The inset graph shows the variation of PPy. It is obvious from the fig. that dielectric loss also follows the same trend as dielectric constant. The decrease in dielectric loss $\epsilon^{\prime \prime}$ with frequency can be attributed to the fact that, at low frequencies, the value of $\epsilon^{\prime \prime}$ is due to the free charge motion within the materials. At moderate frequencies $e^{\prime \prime}$ is due to contribution of ion jumps, conduction loss of ion migration and ion's polarization loss, At high frequencies, the vibration of ions may be the only source of loss and hence dielectric loss has minimum value [13]. On addition of nanoparticles increase in the lower frequency region reflect the enhancement of mobility of charge carrier, with rise in frequency in low frequency region 

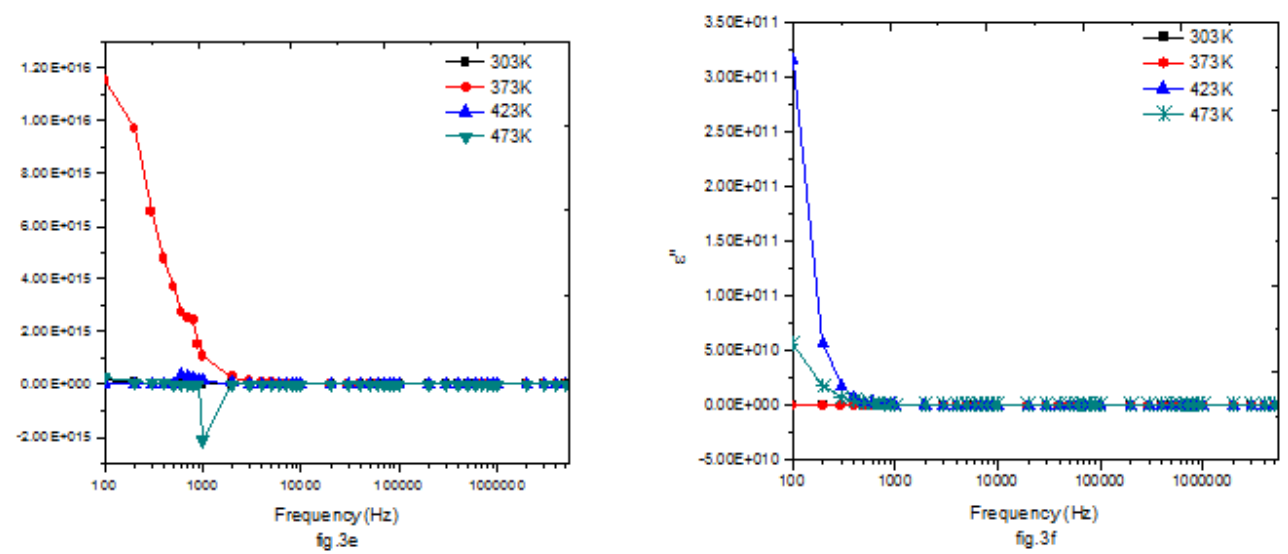

followed by a peak in the loss spectra. The loss peak is shifting towards lower frequency side on addition of nanoparticles.

Dielectric loss peaks are observed at $300 \mathrm{~Hz}$ for 10 and $30 \%$ nanocomposites and at $200 \mathrm{~Hz}$ for $40 \%$ nanocomposites, As the material approaches a relaxation point dielectric loss factor and hence the conductivity increases, this corresponds to drop in dielectric constant. The material is becoming less of energy store and more of energy dissipater, which is related to polarisation characteristics of the material with applied frequency. The relaxation time is calculated by using relation

$$
\tau=\frac{1}{2 \pi f_{m}}
$$

Where $f_{m}$ is the frequency corresponding to maximum energy loss .

The relaxation times at room temperatures ranges from $5.3 \times 10^{-4} \mathrm{~s}$ to $7.9 \times 10^{-4} \mathrm{~s}$ for all nanocomposites

Fig. 3a, 3b, 3c, 3d, 3e and $3 \mathrm{f}$ represents temperature dependence of dielectric loss for nanocomposites of 10, 20,30,40,50 wt.\% and pure Ppy respectively.

At $373 \mathrm{~K}$, Dielectric loss peaks are in the range $200-800 \mathrm{~Hz}$ for all nanocomposites. The relaxation times ranges from $1.59 \times 10^{-4} \mathrm{~s}$ to $7.9 \times 10^{-4} \mathrm{~s}$. At $423 \mathrm{~K}$, dielectric loss peaks are observed at frequencies 200 $7000 \mathrm{~Hz}$. The relaxation times ranges from $2.2 \times 10^{-5}$ to $7.95 \times 10^{-4} \mathrm{~s}$. At $473 \mathrm{~K}$, Dielectric loss peaks are observed at frequencies $200-60000 \mathrm{~Hz}$. The relaxation times ranges from $2.65 \times 10^{-6} \mathrm{~s}$ to $3.97 \times 10^{-4} \mathrm{~s}$. It is clear that the relaxation time decreases with increase of temperature and this may be due to the increase in grain size than the grain boundary[14].

\subsection{Tan $\delta$ (Tangent Loss)}
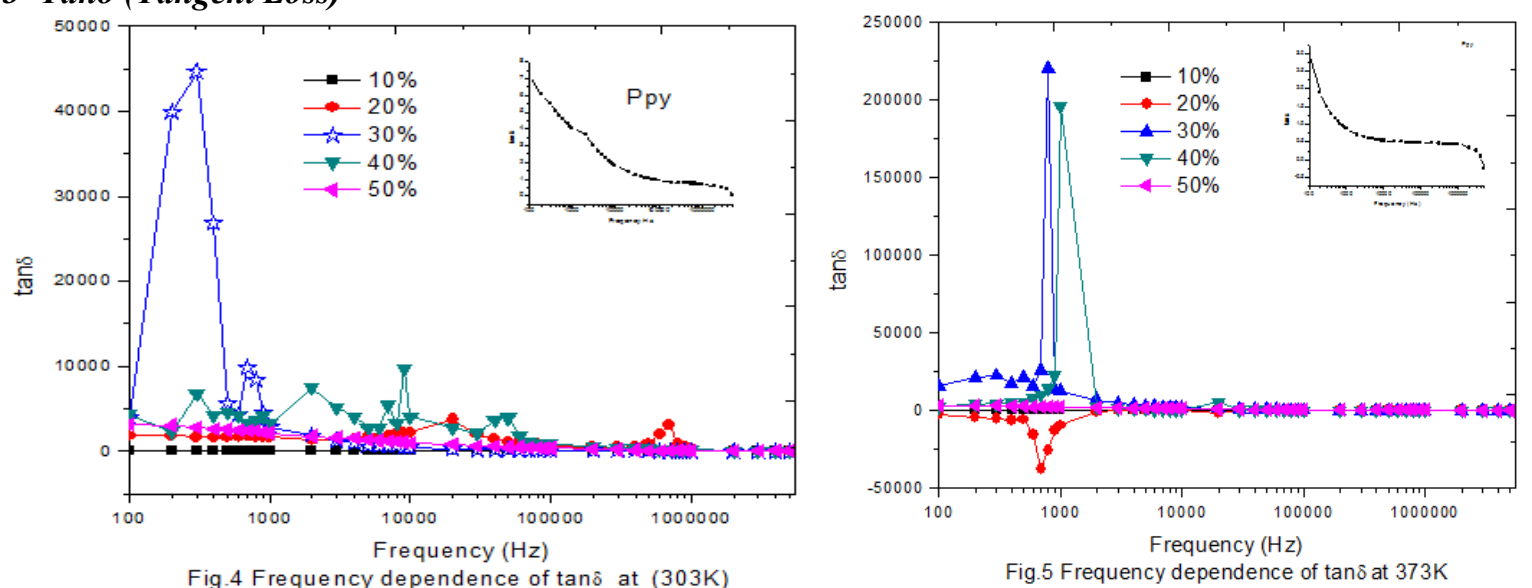

Fig.5 shows the variation of tangent $\operatorname{loss}(\tan \delta)$ with frequency for nanocomposites of different compositions at room temperatures. The loss spectra characterized by peak appearing at a characteristic frequency for all nanocomposites suggest the presence of relaxing dipoles in all the samples. The strength and frequency of relaxation depend on characteristic property of dipolar relaxation.

The tangent loss peaks shift towards the lower frequency side, on addition nanomaterial it is believed that there is an increase in the crystalline content in the materials. It is evidenced by the peak shifting towards 
lower frequency side, thereby increasing the relaxation time. But with increase in temperature loss peaks are shifted towards higher frequency indicating decrease in relaxation time

The Dielectric constant $\left(\varepsilon^{\prime}\right)$, dielectric loss $\left(\epsilon^{\prime \prime}\right)$, and tan $\delta$ for $1 \mathrm{KHz}, 10 \mathrm{KHz}, 100 \mathrm{KHz}$ and $1 \mathrm{MHz}$ at, $303 \mathrm{~K}$ and $373 \mathrm{~K}$ are compared in Table 1 and Table 2 respectively..

Table 1. Dielectric parameters of nanocomposites at $303 \mathrm{~K}$

\begin{tabular}{|c|c|c|c|c|c|c|}
\hline Sample $\rightarrow$ & \multicolumn{2}{|c|}{$10 \%$} & & \multicolumn{3}{|l|}{$20 \%$} \\
\hline Frequency & $\varepsilon^{\prime}$ & $\epsilon^{\prime \prime}$ & $\tan \square$ & $\varepsilon^{\prime}$ & $\epsilon^{\prime \prime}$ & $\tan \square$ \\
\hline $1 \mathrm{KHz}$ & 5023.10 & 8952.98 & 1.7823 & $2.949 \times 10^{10}$ & $4.86 \times 10^{13}$ & 1649.82 \\
\hline $10 \mathrm{KHz}$ & 1168.79 & 364.49 & 0.311 & $3.360 \times 10^{9}$ & $7.382 \times 10^{12}$ & 2197.01 \\
\hline $100 \mathrm{KHz}$ & 936.40 & 82.036 & 0.0876 & $1.007 \times 10^{8}$ & $6.925 \times 10^{10}$ & 687.294 \\
\hline \multirow[t]{2}{*}{$1 \mathrm{MHz}$} & 867.974 & 26.576 & 0.0306 & $-4.13 \times 10^{6}$ & $-1.29 \times 10^{9}$ & 312.297 \\
\hline & \multicolumn{3}{|c|}{$30 \%$} & \multicolumn{3}{|c|}{$40 \%$} \\
\hline $1 \mathrm{KHz}$ & $3.565 \times 10^{8}$ & $9.991 \times 10^{11}$ & 2802.03 & $-2.55 \times 10^{10}$ & $-8.24 \times 10^{13}$ & 3229 \\
\hline $10 \mathrm{KHz}$ & $5.210 \times 10^{6}$ & $2.321 \times 10^{9}$ & 445.594 & $6.99 \times 10^{9}$ & $2.870 \times 10^{13}$ & 4105 \\
\hline $100 \mathrm{KHz}$ & 83191.04 & $5.973 \times 10^{6}$ & 71.80 & $6.635 \times 10^{7}$ & $5.498 \times 10^{10}$ & 828 \\
\hline \multirow[t]{2}{*}{$1 \mathrm{MHz}$} & 1640.22 & 19718.71 & 12.021 & $1.913 \times 10^{6}$ & $3.589 \times 10^{8}$ & 187 \\
\hline & \multicolumn{3}{|c|}{$50 \%$} & & \multicolumn{2}{|l|}{ Ppy } \\
\hline $1 \mathrm{KHz}$ & $3.412 \times 10^{9}$ & $7.249 \times 10^{12}$ & 2124.62 & 23078.7618 & 92333.371 & 4.000 \\
\hline $10 \mathrm{KHz}$ & $1.613 \times 10^{8}$ & $1.616 \times 10^{11}$ & 1001.46 & 1341.9133 & 2419.236 & 1.802 \\
\hline $100 \mathrm{KHz}$ & $5.393 \times 10^{6}$ & $1.815 \times 10^{9}$ & 336.585 & 230.105 & 210.827 & 0.916 \\
\hline $1 \mathrm{MHz}$ & $1.024 \times 10^{5}$ & $6.655 \times 10^{6}$ & 64.94 & 78.550 & 57.00 & 0.725 \\
\hline
\end{tabular}

Table 2. Dielectric parameters of nanocomposites at $373 \mathrm{~K}$

\begin{tabular}{|c|c|c|c|c|c|c|}
\hline Sample $\rightarrow$ & \multicolumn{3}{|c|}{$10 \%$} & \multicolumn{3}{|l|}{$20 \%$} \\
\hline Frequency & $\varepsilon^{\prime}$ & $\epsilon^{\prime \prime}$ & $\tan \square$ & $\varepsilon^{\prime}$ & $\epsilon^{\prime \prime}$ & $\tan \square$ \\
\hline $1 \mathrm{KHz}$ & -22.068 & 75.128 & -3.404 & $-1.76 \times 10^{12}$ & $1.68 \times 10^{16}$ & -9547.9 \\
\hline $10 \mathrm{KHz}$ & 8.8174 & 0.3597 & 0.0408 & $-2.66 \times 10^{9}$ & $2.83 \times 10^{11}$ & -106.36 \\
\hline $100 \mathrm{KHz}$ & 4.4745 & 0.0807 & 0.0108 & $2.03 \times 10^{7}$ & $-2.87 \times 10^{8}$ & -14.152 \\
\hline \multirow[t]{2}{*}{$1 \mathrm{MHz}$} & 6.8285 & 0.2431 & 0.0356 & $1.78 \times 10^{5}$ & $-1.69 \times 10^{5}$ & -0.1298 \\
\hline & \multicolumn{3}{|c|}{$30 \%$} & \multicolumn{3}{|c|}{$40 \%$} \\
\hline $1 \mathrm{KHz}$ & $7.84 \times 10^{9}$ & $9.91 \times 10^{13}$ & 12631 & $8.14 \times 10^{12}$ & $1.59 \times 10^{18}$ & 195650 \\
\hline $10 \mathrm{KHz}$ & $9.84 \times 10^{7}$ & $1.69 \times 10^{11}$ & 1717.4 & $-4.735 \times 0^{9}$ & $2.37 \times 10^{12}$ & 500.8 \\
\hline $100 \mathrm{KHz}$ & $1.62 \times 10^{6}$ & $4.64 \times 10^{8}$ & 285.52 & $4.37 \times 10^{7}$ & $2.48 \times 10^{9}$ & 56.72 \\
\hline \multirow[t]{2}{*}{$1 \mathrm{MHz}$} & $2.01 \times 10^{4}$ & $7.28 \times 0^{5}$ & 36.17 & $3.78 \times 10^{5}$ & $2.02 \times 10^{6}$ & 5.33 \\
\hline & \multicolumn{3}{|c|}{$\mathbf{5 0 \%}$} & \multicolumn{3}{|c|}{ Pрy } \\
\hline $1 \mathrm{KHz}$ & $7.95 \times 10^{10}$ & $1.07 \times 10^{15}$ & 13490 & 197.366 & 172.360 & 0.8733 \\
\hline $10 \mathrm{KHz}$ & $2.20 \times 10^{9}$ & $1.07 \times 10^{13}$ & 4718.17 & 77.3523 & 41.7355 & 0.5395 \\
\hline $100 \mathrm{KHz}$ & $3.42 \times .10^{9}$ & $2.41 \times 10^{10}$ & 706.31 & 38.390 & 18.319 & 0.477 \\
\hline $1 \mathrm{MHz}$ & $4.82 \times 10^{5}$ & $5.01 \times 10^{7}$ & 103.83 & 19.682 & 8.804 & 0.447 \\
\hline
\end{tabular}

\subsection{Ac Conductivity}

The behaviour of AC conductivity has been studied in the temperature range from room temperature $303 \mathrm{~K}$ to $473 \mathrm{~K}$. Fig.6 shows the variation of conductivity with frequency from $100 \mathrm{~Hz}-5 \mathrm{MHz}$ for all nanocomposites. Fig.7 shows the variation of conductivity with frequency from $100 \mathrm{~Hz}$ to $5 \mathrm{MHz}$ for nanocomposites of 50wt.\%. The AC conductivity is almost constant from $100 \mathrm{~Hz}-1 \mathrm{MHz}$ beyond this frequency it decreases, the highest conductivity is observed at $373 \mathrm{~K}$. The same behaviour of conductivity was observed for other studied nanocomposites, except for $10 \%$ which do not show any variation at higher temperature. As shown in Fig, two trends appeared, the first one is frequency independent conductivity which is due to dominating DC conductivity component, where the conductivity is contributed by free charges available in the composite system, and another trend in which conductivity is frequency dependent where AC conductivity decreases due to trapping of charge carrier hop or due to blocking of conduction path by insulating $\mathrm{CuZnFe}_{2} \mathrm{O}_{4}$ nanoparticles. The increase in $\mathrm{AC}$ conductivity with temperature observed at $373 \mathrm{~K}$ may be attributed to increase in hopping rate[15]. 


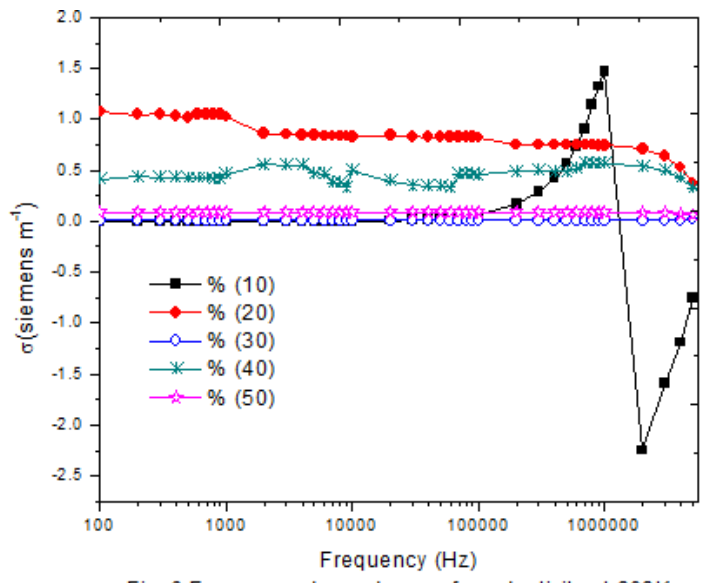

Fig. 6 Frequency dependence of conductivity at $303 \mathrm{~K}$

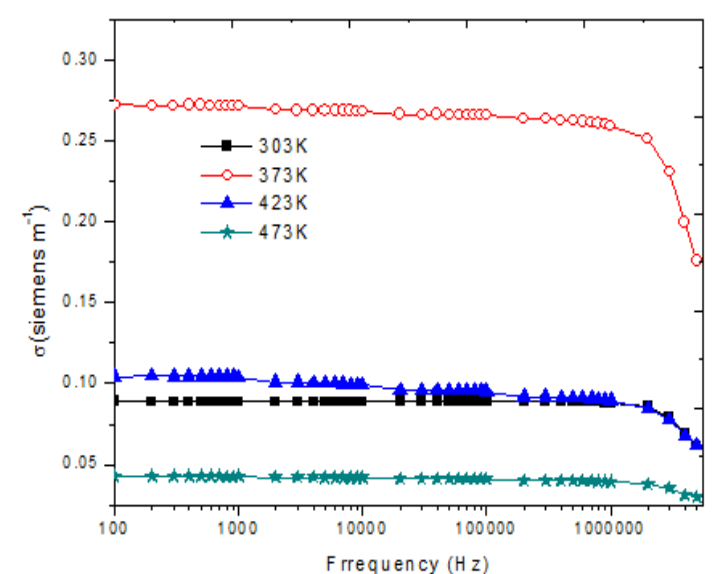

Fig. 7 Temperature dependence of conductivity of nanocomposites $-50 \%$

\section{Conclusions}

The AC conductivity parameters - dielectric constant, dielectric loss and tan $\delta$ were measured by using impedance analyzer. The values of the dielectric constant and dielectric loss were found to decrease with frequency and increase with temperature. The dielectric loss peaks are observed at lower frequency side at room temperature but shifted towards higher frequency side at higher temperature indicating decrease in relaxation time, The relaxation time was found to be in the range $10^{-6}$ to $10^{-4} \mathrm{~s}$. The AC conductivity was found to be almost independent of frequency upto $1 \mathrm{MHz}$, beyond this frequency decrease in conductivity was observed. The highest conductivity was observed at $373 \mathrm{~K}$ for all nanocomposites. The significant increase in dielectric constant makes them a potential candidate for dielectrics in capacitors. In the area of passive components, high dielectric materials are finding increased application in capacitors for decoupling, timing, filtering, and many other functions. Passive components, including capacitors, usually out- number active components in rapidly growing applications such as cell phones, global positioning systems, receivers, and other wireless devices. Due to the negative dielectric constant they can be used in metamaterials and NIMs which are used in unique optical and microwave applications,

\section{Acknowledgements}

The authors would like to acknowledge Dr.Maliikarjuna Babu, Principal, B.M.S. College of Engineering Bangalore and Dr. Pranesh, HOD, Dept. of Physics, B.M.S.C.E., Bangalore for their support in carrying out this work.

\section{References}

[1] Komillasuri, S. Annapoorni and R P Tandon Phase change induced by polypyrrole in iron-oxide polypyrrole nanocomposite, Bull. Mater. Sci., Vol.24, No. 6. pp. 563-567. (2001)

[2] Hamed arami, Mahyar Mazloumi,Razieh Khalifehzadeh,Shahriar Hojjati Emami,S.K.Sadrnezhaad, Polypyrrole/Multiwall carbon nanotube nanocomposites polymerized on copper substrates, Materials Letters. Vol. 61, 4412-4415. (2007)

[3] Lina Mikoliunaite, Reda Kubiliute, Anton Popov, Jaroslav Voronovi, Simas Sakirzanovas, Almira Ramanaviciene, Arunas Ramanavicius, Development of Gold nanoparticles. chemija. vol. 25. No. 2 ,P.63-69. (2014)

[4] J. Jancar, J.F.Douglas, F.W.Starr, S.K.Kumar, P.Cassagnau, A.J.Lesser, S.S.Sternstein M.J.Buehler; Current issues in research on structure-property relationships in polymer nanocomposites, polymer. Volume $51,3321-3343$. (2010)

[5] Ritu P. Mahare, Devendra K. Burghate, Subhash B. Kondawar, Development of nanocomposites based on polypyrrole and carbon nanoyubes for supercapacitors, Adv.Mat.Lett.,.5(7) 400-405. (2014)

[6] Anuj Jain,1 Ravi Kant Baranwal,1 Ajaya Bharti,1 Z. Vakil,1 and C. S. Prajapati2, Study of Zn-Cu Ferrite Nanoparticles for LPG Sensing, The Scientific World Journal Volume 2013, Article ID 790359, 7 pages , (2013)

[7] V.S. Shanthala S.N. Shobha Devi , M. V. Murugendrappa, Optical band gap Studies of Polypyrrole doped with CuZnFe2O4 nano particles, International Journal of Scientific and Research Publications, Volume 6, Issue 9, , 21-26, (2016)

[8] M.V. Murugendrappa and Ambika Prasad, Chemical Synthesis Characterization and dc Conductivity of polypyrrole $-\gamma-\mathrm{Fe}_{2} \mathrm{O}_{3}$ composites , J of Applied Polymer Science. 103, 2797. (2007)

[9] Dillip K. Pradhan, R. N. P. Choudhary, B. K. Samantaray, Studies of Dielectric Relaxation and AC Conductivity Behavior of Plasticized Polymer Nanocomposite Electrolytes, Int. J. Electrochem. Sci., 3 , pp 597 - 608, (2008)

[10] D.K.Ray, A.K. Himanshu \& T.P Sinha, Structural and low frequency dielectric studies of conucting polymer nanocomposites, Indian journal of pure \& Applied PhysicsVol.45, pp.692-699. ( 2007),

[11] Balbir Singh Patil,Neha,Jai Prakash,Rajesh Kumar, S.K. Tripathi, Nagesh Thakur, Dielectric Properties and AC Conductivity Measurements of Amorphous Ge15Se85Glass, Journal of Nano and electronic Physics, Vol.5 no.2, 02019 (4pp), (2013)

[12] H.M. El-Mallah, AC Electrical Conductivity and Dielectric Properties of Perovskite (Pb,Ca)TiO3 Ceramic, Acta Physica Polonica a, Vol. 122, 174-179, (2012)

[13] K.PraveenKumar, T.Shankarappa, J.S.Ashwajeet and R.Ramana, Dielectric and AC Conductivity Studies in Ppy-Ag Nanocomposites, Journals of Polymers, Volume 2015 Article ID 893148,5 pages. (2015) 
[14] Salma M Shaban, Bushta S Mahdi \& Rad MS AI-Haddad, AC conductivity and dielectric behaviour of $\mathrm{CuZnSnO}_{4}$ compound prepared by powder technology Indian Journal of Pure \& Applied Physics Vol.51 ,pp 784-787. (2013)

[15] A.Kumar and S.Sarmah,. AC conductivity and direlectric nspectroscopc studies of polypyrrole-titanium dioxide hybrid nanocomposites, Phy.Status Solidi A208,No.9, 2203-2210 , (2011) 